\title{
O potencial do processo de fotocatálise heterogênea consorciado à adsorção para o tratamento de água com Microcystis aeruginosa e seus metabólitos
}

\section{Potential of the heterogeneous photocatalysis process consortium to the adsorption for the treatment of water with Microcystis aeruginosa and its metabolites}

Data de entrada: 15/07/2019

Data de aprovação: $18 / 11 / 2019$

Andréa Maria Brandão Mendes de Oliveira ${ }^{1 *}$ | Luiz Fernando de Oliveira Coelho'

Eduarda Bezerra Lima de Almeida ${ }^{2}$ | Antonia Samylla Oliveira Almeida ${ }^{2}$ |

Marianna Correia Aragão ${ }^{2}$ | José Capelo Neto

DOI: https://doi.org/10.36659/dae.2021.006

\section{ORCID ID}

Oliveira AMBM (ID) https://orcid.org/0000-0003-2313-1508

Coelho LFO (D) https://orcid.org/0000-0002-4680-1634

Almeida EBL (D) https://orcid.org/0000-0003-1599-7395
Almeida ASO (D) https://orcid.org/0000-0002-6665-8257

Aragão MC (D) https://orcid.org/0000-0001-9249-6805

Capelo NetoJ (D) https://orcid.org/0000-0002-7669-7153

\section{Resumo}

Os processos de fotocatálise heterogênea e adsorção são apontados como alternativas economicamente favoráveis para remoção de contaminantes em água e fazem parte de uma das áreas mais intensamente investigadas devido ao caráter promissor de suas aplicações. Porém, como o gargalo da aplicação tecnológica da fotocatálise heterogênea é a retenção e a reutilização do fotocatalisador dióxido de titânio, faz-se ainda necessário estudar técnicas para sua imobilização em suportes sólidos. Este artigo tem como objetivo apresentar o potencial do processo de fotocatálise heterogênea consorciado à adsorção para o tratamento de água contendo Microcystis aeruginosa e seus metabólitos. $\mathrm{O}$ uso de argilominerais se mostra bastante promissor nas áreas de adsorção e catálise voltadas para o saneamento ambiental.

Palavras-chave: Microcistinas. POA. Cianobactérias. Argilomineral.

\section{Abstract}

The heterogeneous photocatalysis and adsorption processes are considered economically favorable alternatives for removal of contaminants in water, and they are part of one of the most intensely investigated areas due to the promising character of their applications. However, as the difficult of the heterogeneous photocatalysis technological application is the retention and reuse of the photocatalyst titanium dioxide $\left(\mathrm{TiO}_{2}\right)$, it is still necessary to study techniques for its immobilization in solid supports. This article aims to present the potential of the heterogeneous photocatalysis and adsorption process in a consortium for the treatment of water containing Microcystis aeruginosa and its metabolites. The use of clay minerals is very promising in the areas of adsorption and catalysis for environmental sanitation. In addition, clay minerals are abundant in nature, resulting in low cost in their use and encouraging new research aimed at their use in water treatment.

Keywords: Microcystins. AOPs. Cyanobacteria. Clay minerals.

\footnotetext{
${ }^{1}$ Universidade Federal de Campina Grande - Campina Grande - Paraíba - Brasil.

2 Universidade Federal do Ceará - Fortaleza - Ceará - Brasil.

* Autora correspondente: prof.andreabrandaoagmail.com.
} 


\section{INTRODUÇÃO}

A captação de água para abastecimento humano, na maioria das regiões do planeta, é realizada em mananciais superficiais que são passíveis de contaminação por diversos agentes. Um desses agentes são as cianobactérias, microrganismos encontrados em grande parte das águas de superfície e potencialmente produtores de metabólitos secundários; dentre eles têm-se as toxinas e os compostos que atribuem sabor e odor (MIB e geosmina). Ademais, diante do problema da seca, a qual está majoritariamente localizada no Nordeste do Brasil, tem-se uma maior concentração de nutrientes nos reservatórios artificiais e naturais, possibilitando uma maior proliferação de cianobactérias. Dentro disso, faz-se necessário um olhar atencioso voltado para o desenvolvimento de novos métodos de tratamento das águas utilizadas para abastecimento. Dentre os diversos processos de tratamentos existentes, a adsorção tem sido largamente utilizada para a remoção de diversos poluentes presentes na água, em especial aqueles que não são facilmente biodegradáveis.

O processo de adsorção é considerado uma operação de transferência de massa, uma vez que um constituinte, denominado adsorvato, é transferido da fase líquida para uma fase sólida (adsorvente) (TCHOBANOGLOUS et al., 2016). Na busca de um processo de adsorção economicamente viável e eficiente, no tratamento aquoso por exemplo, várias pesquisas têm avaliado diferentes materiais que possam ser utilizados como adsorventes. Dentre esses, os argilominerais apresentam-se como bastante promissores, uma vez que possuem alta disponibilidade, custo significativamente baixo, propriedades de adsorção elevada, não toxicidade e grande potencial de troca iônica (DOGAN, ALKAN e KARAOGLU, 2010). Dessa forma, muitos são os trabalhos que apresentam resultados positivos sobre a utilização de argilominerais em processos de adsorção de cianobactérias e cianotoxinas (COUTO et al. 2013; CHANG et al. 2017; WANG et al. 2014; GU et al. 2016; LIU, WALKER e LENHART, 2019; MORRIS et al. 2000).

A utilização de processos combinados para a descontaminação ambiental pode apresentar vantagens sobre processos simples em sistemas de tratamento. A combinação de processos oxidativos avançados (POAs) utilizando peróxido de hidrogênio, ozônio, luz ultravioleta e $\mathrm{TiO}_{2}$, tem sido testada recentemente como alternativa aos processos de tratamento como a adsorção com resultados promissores. A fotocatálise heterogênea apresenta potencial de aplicação como método de descontaminação em fase aquosa, considerando vários fatores que vão desde sua eficiência até o custo envolvido no processo (ARAÚjO et al. 2016).

A degradação de compostos orgânicos por meio de fotocatálise heterogênea, assim como os princípios que fundamentam o processo, têm sido bastante documentados (ANTONIOU et al., 2018; WAN et al., 2016). No tratamento de águas contendo poluentes orgânicos, os radicais hidroxila gerados na etapa inicial e nas reações de propagação podem desempenhar um papel fundamental na degradação de poluentes. Devido ao seu alto poder oxidante, os radicais $\cdot \mathrm{OH}$ podem agir como eletrófilos em reações com compostos orgânicos, atacando as regiões ricas em elétrons e promovendo a degradação dos mesmos (STEFAN, 2018). A fotocatálise heterogênea possui capacidade de reagir tanto com as espécies microbianas (ação desinfetante) como com seus metabolitos orgânicos. A técnica de fotocatálise pode ser utilizada para o (pré)tratamento de águas para abastecimento visando diminuir a quantidade de cianobactérias e suas toxinas e diminuir os riscos à saúde pública (BRITO, 2013). As microcistinas são uma família de toxinas produzidas por espécies de cianobactérias, prin- 
cipalmente pela Microcystis aeruginosa, mas também por outros gêneros, como Anabaena, Nodularia, Oscillatoria, Nostoc e Cylindrospermopsis (LEAL \& SOARES, 2004). As estruturas das microcistinas são determinadas como monocíclicas, e fazem parte de sua composição dois L-aminoácidos variáveis que incluem, por exemplo, leucina e alanina (LA), leucina e arginina (LR), tirosina e arginina (YR), tirosina e alanina (YA), tirosina e metionina (YM), e dois aminoácidos não usuais, Nmetildehidroalanina (Mdha) e ácido 3-amino-metoxi-10fenil-2,6,8-trimetildeca-4,6-dienóico (Adda). Sua toxicidade é classificada em 3 grupos, dependendo da substituição de dois L-aminoácidos variáveis: forte: -LR, -LA, e -YR; média: -WR; ou fraca: -RR e -M(O)R. Resultados de pesquisas sugerem que os resíduos de Adda e Glu da molécula de microcistina-LR possuem importante papel na hepatotoxidade da microcistina (LEAL \& SOARES, 2004).

Para garantia da saúde pública, tem sido estudada a aplicação de tecnologias complementares àquelas do tratamento do ciclo completo das ETAs. Com isso, este trabalho tem como objetivo despertar na comunidade científica da área de saneamento ambiental a potencialidade do processo de fotocatálise heterogênea consorciado à adsorção para tratamento de água com Microcystis aeruginosa e seus metabólitos.

\section{PROCESSOS DE REMOÇÃO DAS MICROCISTINAS EM ÁGUA}

As cianotoxinas podem ser encontradas sob duas condições: intracelular, ou seja, ainda não foi liberada para o meio, e extracelular, a qual está localizada exteriormente à célula da cianobactéria. Assim, dependendo de onde elas se encontrem há diferentes mecanismos para a remoção das mesmas. Para a remoção de cianotoxina intrace- lular, utilizam-se os seguintes sistemas convencionais de tratamento: ciclo completo, filtração direta, processos com membrana e flotação com ar dissolvido. Já entre os métodos que são capazes de remover toxinas extracelulares têm-se: cloração, osmose reversa, processos oxidativos avançados e adsorção por carvão ativado (PINHO et al. 2015). A utilização de carvão ativado em pó (CAP) faz-se menos vantajosa devido ao fator econômico e, ainda, ao fato de que no fim do processo há uma geração excessiva de lodo. Com relação à utilização de carvão ativado granular, estudos indicam que provoca uma diminuição na capacidade de remoção quando há a presença de matéria orgânica natural (CHESTNUTT, BACH e MAZYCK, 2007; WANG et al. 2017). Em contrapartida, processos oxidativos avançados utilizando a fotocatálise heterogênea possuem a capacidade de degradar moléculas orgânicas, até mesmo as mais estáveis, a partir da produção de espécies oxidativas (radical hidroxila, $0 \cdot 2$, HO·2) (RAMÍREZ \& GALEANO, 2017).

\subsection{Fotocatálise heterogênea para remediação das microcistinas}

Com o intuito de elucidar os possíveis mecanismos de degradação da microcistina-LR, assim como analisar os subprodutos gerados, Liu et al. (2003) caracterizaram estruturalmente os resultados da oxidação fotocatalítica da cianotoxina supracitada. Com isso, obtiveram que os principais mecanismos envolvidos são: a isomerização geométrica causada pela radiação UV; a substituição e a clivagem da estrutura de dieno conjugada de Adda como ilustra a Fig. 1, causadas pelo ataque do radical hidroxila e posterior oxidação obtendo a eliminação da toxicidade não somente da microcistina-LR como também de seus subprodutos. 


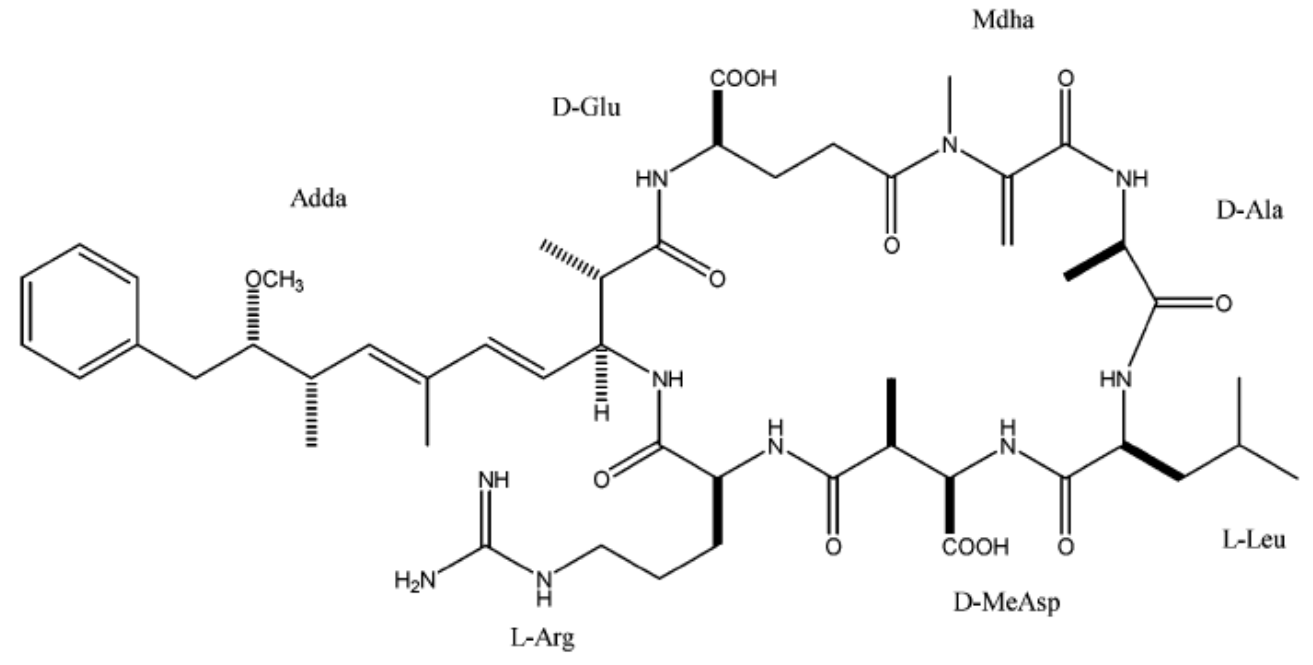

Figura 1 - Dieno conjugada de Adda.

Fonte: Liu et al. 2003.

Ainda que a prevalência dos trabalhos encontrados na literatura seja o uso de dióxido de titânio, há outros semicondutores, como o óxido de cobre e o óxido de zinco, dentre outros. Na Tabela 1 apresentam-se alguns trabalhos desenvolvidos utilizando catalisadores, assim como os resultados obtidos.

Tabela 1 - Fotocatálise heterogênea para a remoção de microcistinas.

\begin{tabular}{|c|c|c|}
\hline Autor & Descrição dos resultados & Materiais \\
\hline Pinho et al. (2015) & $\begin{array}{c}\text { Avaliou-se a capacidade da destruição celular da Microcystis aeruginosa, além da degradação } \\
\text { de microcistina-LR e a cilindrospermopsina. A matéria orgânica presente na água natural } \\
\text { ocasionou uma baixa taxa de degradação da microcistina-LR, devido a uma possível } \\
\text { competição por fótons UV. Para uma degradação de } 80 \% \text { da microcistina-LR, foram } \\
\text { necessárias aproximadamente } 3,5 \text { horas. }\end{array}$ & $\begin{array}{c}\text { Dióxido de titânio (Degussa P25) } \\
\text { sob radiação solar }\end{array}$ \\
\hline Zou et al. (2016) & $\begin{array}{l}\text { Utilizando-se a precipitação-fotorredução para depositar nanopartículas de } \mathrm{Ag} / \mathrm{AgCl} \text { no } \\
\text { semicondutor niobato de potássio (K6Nb10.8O30), foi avaliada a capacidade fotocatalítica } \\
\text { do composto ( } \mathrm{Ag} / \mathrm{AgCl} \text { - K6Nb10.8030) para a degradação da microcistina-LR sob luz visível. } \\
\text { Para fins comparativos, foi utilizado o semicondutor } \mathrm{K} 6 \mathrm{Nb} 10.8 \mathrm{O} 30 \text { em sua forma pura, para } \\
\text { então observar sua atividade fotocatalítica. A partir dos resultados, foi possível observar que } \\
\text { o semicondutor puro exibiu taxa de degradação muito menor, aproximadamente } 20 \% \text { após } 1 \\
\text { hora de exposição à luz visível, enquanto } \mathrm{Ag} / \mathrm{AgCl}-\mathrm{K} 6 \mathrm{Nb} 10.8 \mathrm{O} 30 \text { apontou uma deterioração } \\
\text { de aproximadamente } 100 \% \text { após } 1 \text { hora de procedimento. }\end{array}$ & $\begin{array}{l}\text { Niobato de potássio + } \\
\mathrm{Ag} / \mathrm{AgCl} \text { sob luz visível }\end{array}$ \\
\hline Antoniou et al. (2018) & $\begin{array}{l}\text { Utilizaram-se duas fontes de radicais de sulfato (persulfato/PS e peroximonossulfato/PMS) } \\
\text { para investigar a eficiência do } \mathrm{TiO}_{2} \text { quanto à fotodegradação da microcistina-LR sob radiação } \\
\text { UV. Obteve-se que o } \mathrm{TiO}_{2}-\mathrm{PMS} \text { apresentou uma taxa de degradação mais rápida do que o } \\
\mathrm{TiO}_{2} \text {-PS, na ordem de } 2: 1 \text {. A porcentagem de remoção da cianotoxina para ambos foi igual a } \\
100 \% \text {, sendo que o primeiro foi após } 5 \text { minutos de radiação UV e o segundo após } 10 \text { minutos. }\end{array}$ & $\begin{array}{l}\text { Persulfato/PS e } \\
\text { peroximonossulfato/PMS } \mathrm{TiO}_{2} \\
\text { sob radiação UV }\end{array}$ \\
\hline Song et al. (2018) & $\begin{array}{l}\text { A eficiência do } \mathrm{TiO}_{2} \text { com nitrato de carbono grafítico em diferentes proporções mássicas na } \\
\text { remoção da microcistina- } \mathrm{LR} \text { com radiação solar foi avaliada. Dentre as diversas razões mássicas } \\
\text { avaliadas, a que apresentou maior performance para remoção da Microcystis aeruginosa, } \\
\text { simultaneamente com a degradação da microcistina- } \mathrm{LR} \text {, foi o valor } 0,03 \mathrm{~g}-\mathrm{C} \mathrm{N} 4 / \mathrm{TiO}_{2} \text {. }\end{array}$ & $\begin{array}{l}\text { Nitrato de carbono grafítico com } \\
\mathrm{TiO}_{2} / \text { sob irradiação solar }\end{array}$ \\
\hline Pestana et al. (2015) & $\begin{array}{l}\text { Avaliaram a capacidade de fotocatálise de esferas de vidro revestidas com dióxido de } \\
\text { titânio }\left(\mathrm{TiO}_{2}\right) \text {, com relação a duas cianotoxinas. } \mathrm{TiO}_{2} \text { em sua fase anatásio foi utilizado } \\
\text { para revestimento das esferas de sílica. A degradação de onze variantes de microcistina e } \\
\text { nodularina, utilizando-se da radiação UV, foi avaliada. A taxa de reação das microesferas foi } \\
\text { tão eficiente quanto o fotocatalisador em pó. Com relação à degradação da microcistina-LR, } \\
\text { alcançou-se, para ambos os materiais, a decomposição da toxina em apenas } 4 \text { minutos. }\end{array}$ & $\begin{array}{l}\text { Microesferas de vidro com } \\
\text { Degussa P25 sob radiação UV }\end{array}$ \\
\hline Gu et al. (2016) & $\begin{array}{l}\text { Utilizaram-se nanopartículas de óxido de cobre } \mathrm{Cu}_{2} \mathrm{O} \text { suportada em montmorilonita, floculante } \\
\text { e fotocatalisador para remoção da Microcystis aeruginosa. A partícula foi preparada pela redução } \\
\text { do } \mathrm{Cu}^{2+} \text { absorvido na montmorilonita, utilizando glicose e etilenoglicol como redutor. Obteve-se } \\
\text { uma remoção de } 90,4 \% \text { de Microcystis aeruginosa em } 3 \text { horas sob luz visível. }\end{array}$ & $\begin{array}{l}\text { Montmorilonita revestida } \\
\text { com } \mathrm{Cu}_{2} \mathrm{O} \text { sob luz visível }\end{array}$ \\
\hline Gu et al. (2015) & $\begin{array}{l}\text { A capacidade fotocatalítica de inibição da cianobactéria Microcystis aeruginosa sob radiação } \\
\text { UV utilizando-se montmorilonita intercalada com óxidos de zinco (ZnO) foi avaliada. Como } \\
\text { resultado, obteve-se uma remoção de } 95 \% \text { de células após } 1 \text { hora de exposição. }\end{array}$ & $\begin{array}{c}\mathrm{ZnO} \text { intercalado em } \\
\text { montmorilonita sob radiação UV }\end{array}$ \\
\hline
\end{tabular}




\section{2 $\mathrm{TiO}_{2}$ destaque como semicondutor}

Diante dos diversos semicondutores existentes, faz-se necessário destacar o dióxido de titânio devido à sua alta fotoatividade quando comparada com outros materiais. Além disso, o mesmo possui propriedades estáveis e é quimicamente inerte, além de ser abundante e possuir baixo custo (DAGHRIR, DROGUI \& ROBERT, 2013). Ademais, o dióxido de titânio tem sido um dos catalisadores mais atrativos devido à sua ação simultânea tanto para remoção de matéria orgânica como inativação microbiana, sem demandar adição de outros agentes oxidantes (GALEANO et al. 2017; RUBIO, CASANUEVA \& NEBOT, 2013).

A maioria dos estudos utilizando $\mathrm{TiO}_{2}$ como fotocatalisador são realizados com o mesmo em suspensão ou sob mistura completa, o que confere vantagens como: maior área superficial disponível para reação e minimização de resistência de transferência de massa. Por outro lado, a principal desvantagem deste método relaciona-se à perda contínua do fotocatalisador e à elevação dos custos operacionais, dada a dificuldade de recuperação do $\mathrm{TiO}_{2}$, uma vez que, em sua forma comercial, apresenta baixa velocidade de sedimentação (BORGES, 2016).

De acordo com Borges (2016), embora haja diversas técnicas para imobilizar o fotocatalisador sobre o suporte, na maioria dos casos tem-se usado ligação térmica e métodos de recobrimento sol-gel. Ainda que diversos fatores influenciem diretamente a efetividade da imobilização, a rugosidade do suporte e a característica da suspensão são considerados os mais relevantes (BORGES, 2016). Os meios suporte devem ser substratos porosos e com grande capacidade de adsorção a fim de promover um aumento na área superficial, uma maior estabilidade térmica na transformação das fases cristalinas e uma diminuição da densidade do catalisador, podendo promover assim uma maior interação fotocatalisador/poluente (BORGES, 2016).

\subsection{Adsorção como etapa simbiótica ao processo de fotocatálise}

O dióxido de titânio em sua forma suspensa, apesar de ser amplamente utilizado para a fotocatálise de contaminantes, possui sua atividade limitada quanto ao tratamento de poluentes que estejam em baixa concentração. Isso se deve à sua baixa porosidade e baixa capacidade de adsorção (BELVER et al. 2017). Ainda, há a difícil remoção/recuperação após o fim do processo (SZCZEPANIK, 2017). Portanto, muitos estudos estão centrados em avaliar diferentes tipos de suporte para o dióxido de titânio (BORGES et al. 2016; XING et al. 2018; SZCZEPANIK, 2017; SRIKANTH et al. 2017). Dentre os mais diversos tipos de suporte, destacam-se: fibra de vidro, argilominerais, polímeros e cerâmicas.

À vista disso, em relação a uma matriz polimérica, deve-se ater aos polímeros que não absorvam energia na região de band-gap, que é a região que ativa a ação do fotocatalisador. Por exemplo, o dióxido de titânio possui uma energia de ativação de 365 nm, logo, não se deve escolher como suporte um polímero que absorva energia nessa região, pois iria promover competição e consequentemente tornar o processo menos eficiente, além de suscitar a degradação do suporte quando irradiado (CURCIO, 2011).

Muitos estudos focam na utilização de esferas de vidro, ou fibra de vidro, como suportes (PESTANA et al. 2015; MIRANDA-GARCÍA et al. 2014; MAROTHU et al. 2014), já que os mesmos são inertes e suportam altas temperaturas de calcinação. Porém, avaliando-se especificamente a capacidade de adsorção dentre os diversos meios suportes, apesar de o vidro ser bastante estudado, quem vem chamando a atenção de muitos cientistas são os argilominerais (BELVER et al. 2017; CHEN, ZHU e WANG, 2014; DENG, ZHANG e GENG, 2018; BARBOSA et al. 2015; LABILLE et al. 2015; PRONINA et al. 2015; RAHMAN et al. 2018; ALSHAMERI, YAN e LEI, 2014; LIU e ZHANG, 2014; SUN et al. 2015; ZHANG et al. 2018). 


\subsection{Argilominerais como adsorvente e suporte para o fotocatalisador $\mathrm{TiO}_{2}$}

Recentes estudos mostram quão benéfica pode ser a utilização de argilominerais como meio suporte, e sua aplicação tem sido diversa quanto à remoção de poluentes, como cobre (DENG, ZHANG e GENG, 2018), corantes (RAHMAN et al. 2018; ZHANG et al. 2018), fosfato (ALSHAMERI, YAN e LEI, 2014), pesticidas (BELVER et al. 2017) e antibióticos (VAIZOĞULLAR, 2017). Diante disso, sabe-se que tais minerais argilosos possuem especificidades essenciais para a capacidade adsortiva, como uma grande área superficial, uma elevada estabilidade química, boas propriedades mecânicas e grandes quantidades de poros. Ainda, faz-se necessário salientar a capacidade das argilas fornecerem uma maior quantidade de sítios ativos superficiais ao se dispersarem partículas de $\mathrm{TiO}_{2}$ (SZCZEPANIK, 2017).

Apesar de todas as argilas possuírem grandes volumes de poros, algumas se fazem mais vantajosas do que outras devido à sua morfologia estrutural, como reportado por Liu, Walker e Lenhart (2019), que avaliaram a capacidade adsortiva da caulinita, da ilita e da montmorilonita. $O$ trabalho citado objetivou a adsorção da microcistina-LR e, dentre os resultados, obteve-se que a montmorilonita mostrou-se mais eficiente. Isso se dá porque sua estrutura interlamelar possui camada em uma proporção de 2:1, enquanto a caulinita e a ilita possuem camadas em proporção 1:1. Ademais, o mesmo trabalho avaliou a capacidade adsortiva em três diferentes pHs $(5,7,9)$ e, também, sob dois distintos sistemas saturados (um com íons de Cálcio ${ }^{2+}$ e outro com íons de Sódio+). Como resultado, para as operações feitas em meio saturado com íons de $\mathrm{Ca}^{2+}$, obteve-se, para a caulinita e a ilita, uma melhor adsorção em pH ácido; já para a montmorilonita, o pH básico fez-se mais eficiente. Ainda, para o meio saturado com íons de $\mathrm{Na}^{+}, \mathrm{o}$ caulim apresentou melhor adsorção quando comparado com a ilita, sendo que tal ordem é invertida ao se utilizarem íons de $\mathrm{Ca}^{2+}$.

\section{MÉTODOS DE SINTETIZAÇÃO DE TiO} Barnard, Zapol e Curtiss (2005) demonstraram que a formação da fase anatase é preferencial, em relação à fase rutilo, somente para partículas com tamanhos inferiores a $12-13 \mathrm{~nm}$, ou seja, excedendo-se esse valor há a tendência de transformação de fase. Com isso, faz-se necessária a utilização de metodologias que permitam controlar o tamanho da partícula final (BOERY et al. 2011). Dentre os métodos existentes, destaca-se a rota sol-gel hidrolítica, já que a mesma é a que se faz amplamente citada na literatura (WANG et al. 2014; WANG et al. 2015; BARBOSA et al. 2015; RAHMAN et al. 2018) e, ainda, possibilita uma boa homogeneidade do produto obtido.

Um outro método, porém menos utilizado, é o dos precursores poliméricos, o qual é uma variante do processo sol-gel, na qual a reação de formação é não hidrolítica, gerando um polímero, onde, após calcinação, ou outro método de eliminação de matéria orgânica, forma-se o óxido de interesse (ARIMA et al. 1996; KAKIHANA e YOSHIMURA, 1999). Tal processo está baseado na capacidade de ácidos orgânicos hidroxicarboxílicos formarem quelatos com vários cátions. Quando um álcool polihidroxi é adicionado a este quelato, sob aquecimento e agitação constantes, ocorre uma reação de poliesterificação. Desse modo, observa-se uma distribuição uniforme e homogênea dos cátions metálicos na rede polimérica (LESSING, 1989). Em seguida, com o aquecimento e eliminação do excesso de solvente, e uma resina altamente viscosa é formada (precursor polimérico). Tal resina é calcinada a aproximadamente $300^{\circ} \mathrm{C}$, para a quebra do polímero, resultando na formação do pó precursor, um material semi-carbonizado de cor escura. Em seguida realiza-se um tratamento térmico em diferentes temperaturas para a eliminação do material orgânico e obtenção da fase desejada (TADOKORO e MUCCILO, 2000). A 
desvantagem desse método é a elevada perda de massa e a formação de agregados na calcinação (LEITE, 1995).

Existe, ainda, o método de precipitação homogênea, no qual os precursores clorados de titânio ou do tetraisopropóxido de titânio reagem com uma base (hidróxido de sódio ou de amônio), formando hidróxido de titânio, o qual é convertido por meio da perda de moléculas de água em $\mathrm{TiO}_{2}$ amorfo, onde, sob diferentes tratamentos térmicos, dá origem às três formas cristalinas conhecidas (MILLS e LE HUNTE, 1997). Todavia, a principal desvantagem desse método se deve à relativa dificuldade no controle do tamanho das partículas, já que precipitações muito rápidas tendem a causar a formação de partículas de $\mathrm{TiO}_{2}$ muito grandes, por aglomeração (CHEN e CHEN, 2003). Entretanto, é um método de baixo custo e prático na preparação dos fotocatalisadores.

\subsection{Método sol-gel}

O processo sol-gel é utilizado na obtenção de cerâmicas, vítreos, ou híbridos orgânico-inorgânico na forma de pós (BRINKER et al. 1988). Neste método, o precursor consiste em um elemento metálico rodeado por vários elementos ligantes, os quais podem ser orgânicos ou inorgânicos. O termo sol é designado para definir uma suspensão de partículas coloidais estáveis em um fluido, enquanto o termo gel está relacionado a uma estrutura rígida de partículas interconectadas que formam uma espécie de rede/matriz (BRINKER e SCHERER, 1990). Diante disso, o processo sol-gel trata da transição da fase sol para um sistema gel, onde o processo é dividido em duas etapas elementares: i) a hidrólise do grupo alcóxido com a formação de grupos reativos; ii) a condensação do grupo reativo, que leva à formação do sol e, posteriormente, à formação do gel (BRINKER e SCHERER, 1990). As reações de hidrólise e de condensação produ- zem interconexões entre os centros metálicos (M), que estão conectados por ligações hidroxi (M-OH-M) ou por oxigênio (M-O-M).

No processo de hidrólise, há a distinção entre os precursores, os quais podem ser não ionizados e ionizados. No caso de um precursor orgânico não ionizado, a hidrólise envolve a ligação do íon $\mathrm{OH}$ - ao metal proveniente do precursor, em uma reação que pode ser representada da seguinte forma (BRINKER e SCHERER, 1990):

$\mathrm{M}-\mathrm{OR}+\mathrm{H}_{2} \mathrm{O} \rightarrow \mathrm{M}-\mathrm{OH}+\mathrm{ROH}$ (hidrólise)

Onde $\mathrm{M}$ representa um metal e $\mathrm{R}$ é um ligante orgânico (pode ser uma alquila ou arila). Um metal parcialmente hidrolisado pode reagir com o próprio precursor não hidrolisado (Eq. 1) ou com outras moléculas parcialmente hidrolisadas numa reação de policondensação (Eq. 2).

$\mathrm{M}-\mathrm{OH}+\mathrm{M}-\mathrm{OR} \rightarrow \mathrm{M}-\mathrm{O}-\mathrm{M}+\mathrm{ROH}(1)$
$\mathrm{M}-\mathrm{OH}+\mathrm{M}-\mathrm{OH} \rightarrow \mathrm{M}-\mathrm{O}-\mathrm{M}+\mathrm{H}_{2} \mathrm{O}(2)$

Para precursores ionizados, os cátions de um metal $M$ atraem as moléculas de água dipolares (ou hidroxilas), resultando em unidades hidrolisadas do tipo $\mathrm{M}\left(\mathrm{H}_{2} \mathrm{O}\right)_{n}{ }^{2+}$ ou $\mathrm{M}(\mathrm{OH})_{n}{ }^{z-n}$ (onde 'n' é o número de unidades de coordenação e ' $z$ ' a carga da espécie hidrolisada) que posteriormente polimerizam (PIERRE, 1991).

A partir de uma visão pragmática, o processo pode ser resumido nas seguintes etapas: inicialmente o sistema é constituído por partículas coloidais dispersas (sol), que resultam da polimerização do monômero; tais partículas se ligam, formando pequenas cadeias ramificadas tridimensionais e regiões de microgel, onde o índice de refração e a densidade são próximos aos da dispersão e, portanto, não ocorre decantação; quando o crescimento dessas regiões estruturadas atinge, aproximadamente, a metade do volume total, o sistema passa a apresentar um comportamento elástico; com isso, as regiões es- 
truturadas crescem conjuntamente, culminando na formação de uma rede que ocupa todo o volume do sistema (BRINKER e SCHERER, 1990).

Para remoção do excesso de solvente, deve-se realizar o processo de secagem. Posteriormente, para favorecer a densificação e a sinterização e, com isso, melhorar as propriedades mecânicas, deve-se ainda realizar um tratamento térmico (SRIKANTH et al. 2017).

A principal vantagem desse método sobre os métodos convencionais é a obtenção de sistemas homogêneos com alto grau de pureza e que podem ser calcinados a temperaturas relativamente baixas e, ainda, permitem a síntese de óxidos metálicos com propriedades bem definidas e controladas (SRIKANTH et al. 2017).

\subsection{Análise dos parâmetros do método sol-gel}

Recentemente muitos trabalhos analisaram diferentes aspectos de preparação do método sol-gel (WANG et al. 2014; WANG et al. 2015; BARBOSA et al. 2015; RAHMAN et al. 2018). Dentre eles, Wang et al. (2014) avaliaram diferentes dosagens de butóxido de titânio IV no preparo de uma mistura feita com diatomita (suporte) para, então, mensurar a capacidade de remoção de um corante. Quanto às dosagens $(0.5 \mathrm{~mL} ; 0.75 \mathrm{~mL} ; 1.0 \mathrm{~mL} ; 1.5 \mathrm{~mL} ; 2.0 \mathrm{~mL}$; $3.0 \mathrm{~mL}$ ), as mesmas foram adicionadas após o preparo, sob agitação durante 30 minutos, de uma suspensão de diatomita dispersa em 14 $\mathrm{mL}$ de etanol e $1 \mathrm{~mL}$ de ácido acético. A mistura resultante foi, inicialmente, agitada durante 12 horas e, posteriormente, secada a $105^{\circ} \mathrm{C}$ por 4 horas e calcinada a $750^{\circ} \mathrm{C}$ por 2 horas. Após
1 hora sob radiação UV (>365 nm), obtiveram, como resultado, uma melhor eficiência de remoção para as dosagens de 1.5 e $2.0 \mathrm{~mL}$, ambas correspondentes a 99,7\%. Curiosamente, a menor eficiência obtida foi pelo composto produzido à dosagem de $3.0 \mathrm{~mL}$, com uma porcentagem de remoção igual a 95.9\%.

Outro parâmetro analisado por Wang et al. (2015) foi a temperatura de calcinação, a qual se realizou nos seguintes valores: $450^{\circ} \mathrm{C} ; 550^{\circ} \mathrm{C} ; 650^{\circ} \mathrm{C}$; $750^{\circ} \mathrm{C} ; 850^{\circ} \mathrm{C} ; 950^{\circ} \mathrm{C}$. A partir da análise de Difração por Raios-X observou-se $100 \%$ da forma anatásio apenas para a temperatura de calcinação igual a $650^{\circ} \mathrm{C}$, enquanto a partir do aumento desta, ou seja, para as demais temperaturas, tal porcentagem foi diminuindo. Por conseguinte, a partir das análises de adsorção e fotodegradação durante 2 horas sob radiação UV, obtevese uma melhor eficiência para temperatura de $750^{\circ} \mathrm{C}(92,6 \%)$, seguida da temperatura $650^{\circ} \mathrm{C}$ $(89,1 \%)$. Faz-se necessário salientar, portanto, que a maior eficácia obtida se procedeu para a parcela de $90,6 \%$ da fase anatásio.

Um outro estudo, também com relação à temperatura de calcinação pelo método sol-gel, foi realizado por Barbosa et al. (2015), onde abordaram a sintetização com a argila caulinita e, a partir disso, avaliaram a degradação de dois corantes (azul de metileno e laranja de metila II), também sob radiação UV (365 nm). As temperaturas em questão, $400^{\circ} \mathrm{C}, 700^{\circ} \mathrm{C}$ e $1000^{\circ} \mathrm{C}$, foram submetidas durante 24 horas e, o precursor de titânio utilizado foi o isopropóxido de Ti(IV). A Fig. 2 representa esquematicamente o método de preparação dos compostos via rota sol-gel. 
$1^{\text {st }}$ step: Synthesis
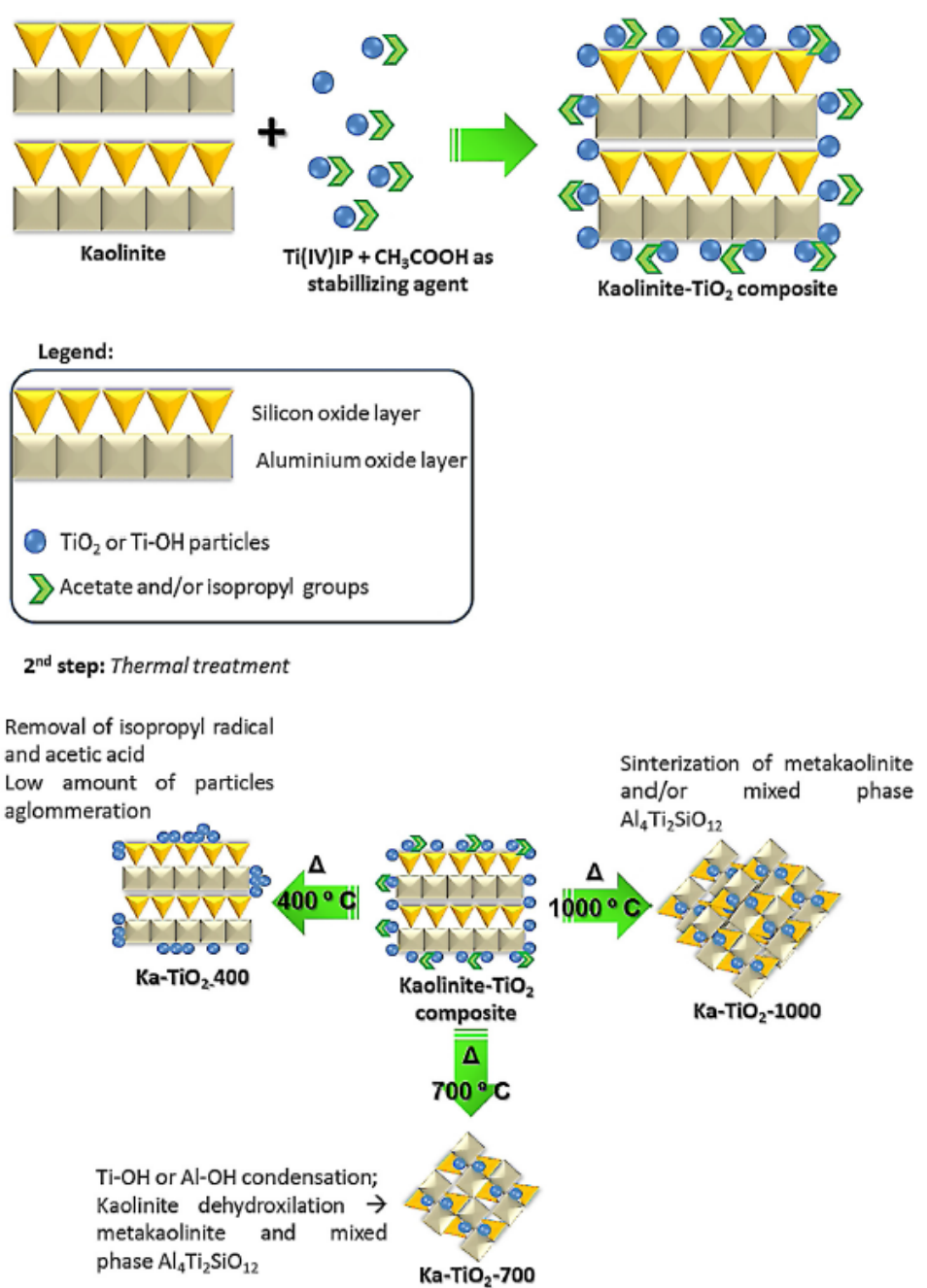

Figura 2 - Esquematização do processo de síntese via rota sol-gel Fonte: Barbosa et al. 2015.

A partir da análise das reações sem a utilização de radiação-UV, após 24 horas, obteve-se uma melhor capacidade de adsorção para o composto que foi calcinado à temperatura de $400^{\circ} \mathrm{C}$ $\left(\mathrm{q}_{\mathrm{e}}=45,89 \mathrm{mg} / \mathrm{g}\right)$, enquanto para as demais temperaturas, $700^{\circ} \mathrm{C}$ e $1000^{\circ} \mathrm{C}$, tais valores diminuíram para $20,05 \mathrm{mg} / \mathrm{g}$ e $5,20 \mathrm{mg} / \mathrm{g}$, respectivamente. Já para as análises de fotodegradação (sob radiação-UV), obtiveram-se semelhantes taxas de degradação para todas as amostras, chegando a $100 \%$ após 1 hora de irradiação.

\subsection{Análises de reutilização}

Com o intuito de avaliar a aplicabilidade industrial dos métodos em questão, faz-se necessário averiguar a eficiência de remoção quanto à reutilização do material. Em vista disso, Deng, Zhang e Geng (2018) avaliaram a remoção de Cobre (II) após 3 ciclos de reutilização do composto, o qual foi produzido com zeólita e $\mathrm{TiO}_{2}$ pelo método hidrotérmico. Obtiveram, após os três ciclos, uma diminuição de 1,2\% na remoção, passando de $100 \%$ no primeiro ciclo para $98,4 \%$ no terceiro ciclo. 
Ainda, Sun et al. (2015) também utilizaram a zeólita como suporte, a qual foi imobilizada com $\mathrm{TiO}_{2}$ a partir do método hidrolítico, e, após 5 ciclos de regeneração, obteve-se uma diminuição de $14,9 \%$ na remoção de $\mathrm{Cr}(\mathrm{VI})$. Onde inicialmente houve uma remoção de $89,9 \%$, ao fim dos ciclos esse valor diminuiu para $75 \%$.

Ainda utilizando o método de deposição hidrolítica para suportar $\mathrm{TiO}_{2}$ em zeólita, Zhang et al. (2018) avaliaram a capacidade de remoção após 5 ciclos de reutilização. Obtiveram, por fim, uma diminuição na capacidade de eliminação do corante Rodamina-B na ordem de $3 \%$, onde o primeiro ciclo apresentou $94 \%$ e o quinto $91 \%$.

Outro estudo que utilizou zeólita como suporte, porém pelo método sol-gel, foi o realizado por Liu, Lim e Amal (2014), o qual objetivou a remoção de ácido húmico. Após 5 repetições, não se obteve diminuição na porcentagem de remoção, a qual passou de $77 \%$ para $80 \%$ ao fim dos ciclos. Tal resultado se contrapõe com o obtido por Borges et al. (2016), o qual também utilizou zeólita como suporte pelo método sol-gel e, após ensaio de erosibilidade, obteve-se uma perda de $1,1 \%$, o que necessariamente levaria a uma diminuição da eficiência fotocatalítica.

O trabalho de Wang et al. (2014) utilizou um método modificado do sol-gel para imobilizar o dióxido de titânio em diatomita. Como resultado, obteve-se uma diminuição, após 5 ciclos, de $99,6 \%$ para $74,7 \%$ na remoção da Rondamina-B.

Ainda com relação ao método sol-gel, o estudo feito por Wang et al. (2017) para remoção de microcistina-LR utilizou perlita expandida como suporte e, ainda, fez-se uso de metais ( $\mathrm{Fe} \mathrm{Ce}$ ) para dopagem do dióxido de titânio. Após 3 ciclos de reúso, observou-se uma diminuição na eficiência fotocatalítica de $97 \%$ para $88 \%$.

\section{CONSIDERAÇÕES FINAIS}

O uso de argilominerais se mostrou bastante propício nas áreas de adsorção e fotocatálise heterogênea de forma consorciada, indicando que é um excelente exemplo da tecnologia moderna. Foi possível observar nesta revisão o quanto o conhecimento da influência das condições experimentais de síntese é importante, pois permite o controle do sistema de modo a se arquitetar as características finais dos materiais obtidos. Devido a essa versatilidade, os materiais podem ser obtidos com diferentes propriedades que permitem sua aplicação nas mais variadas áreas da ciência e da tecnologia, como o apresentado nesta revisão, onde a adsorção utilizando os argilominerais combinados em processo de saneamento ambiental, mais especificamente em tratamento de água na remoção da microcistina mostra-se como uma área muito promissora para os avanços e desafios científicos no tema apresentado nesta pesquisa.

\section{CONTRIBUIÇÃO DOS AUTORES}

Todos os autores contribuíram de forma igualitária.

\section{REFERÊNCIAS}

ALSHAMERI, A.; YAN, C.; LEI, X. Enhancement of phosphate removal from water by TiO2/Yemeni natural zeolite: Preparation, characterization and thermodynamic. Microporous and Mesoporous Materials, v. 196, 2014, p. 145-157.

ANTONIOU, M.G.; BORAEI, I.; SOLAKIDOU, M.; DELIGIANNAKIS, Y.; ABHISHEK, M.; LAWTON, L. A.; EDWARDS, C. Enhancing photocatalytic degradation of the cyanotoxin microcystin-LR with the addition of sulfate-radical generating oxidants. Journal of Hazardous Materials, v. 360, 2018, p. 461-470.

ARIMA, M.; KAKIHANA, M.; NAKAMURA, Y.; YASHIMA, M.; YOSHIMURA, M. Polymerized Complex Route to Barium Titanate Powders Using Barium-Titanium Mixed-Metal Citric Acid Complex. Journal of the American Ceramic Society, v. 79, n. 11, 1996, p. 2847-2856.

BARBOSA, L. V.; MARÇAL, L.; NASSAR, E. J.; CALEFI, P. S.; VICENTE, M. A.; TRUJILLANO, R.; RIVES, V.; GIL, A.; KORILI, S. A.; CIUFFI, K. J.; FARIA, E. H. de. Kaolinite-titanium oxide nanocomposites 
prepared via sol-gel as heterogeneous photocatalysts for dyes degradation. Catalysis Today, v. 246, 2015, p. 133-142.

BARNARD, A. S.; ZAPOL, P.; CURTISS, L. A. Modeling the Morphology and Phase Stability of TiO2 Nanocrystals in Water. Journal of Chemical Theory and Computation, v. 1, 2005, p. 107-116.

BELVER, C.; DIONYSIOU, D. D.; HAN, C.; RODRIGUEZ, J. J. Innovative $\mathrm{W}$-doped titanium dioxide anchored on clay forphotocatalytic removal of atrazine. Catalysis Today, v. 280, 2017, p. 21-28.

BOERY, Mirella Nagib de Oliveira. Obtenção controlada das nanopartículas e das fases cristalinas do dióxido de titânio. 2011. Dissertação (Mestrado em Engenharia Mecânica) - Universidade Estadual de Campinas, Campinas, 2011.

BORGES, S. S.; SILVA, A. C. da; XAVIER, L. P. S.; AQUINO, S. F. de. Imobilização de dióxido de titânio em diferentes materiais suporte para o emprego em fotocatálise heterogênea. Química Nova, v. 39, n. 7, 2016, p. 836-844.

BRINKER, C. J.; Hydrolysis and condensation of silicates: effects on structure. Journal of Non-Crystalline Solids, v. 100, n. 1-3, 1988, p. 31-50.

BRINKER, C. J.; SCHERER, G. The Physics And Chemistry Of Sol-Gel Processing. Boston: Academic Press, 1990.

BRITO, Cristiano Régis Freitas de. Fotocatálise heterogênea associada à adsorção em cerâmica modificada para remoção de ciprofloxacino. 2013. Dissertação (Mestrado em Engenharia Civil) - Universidade Federal do Ceará, Fortaleza, 2013.

CHANG, S.C.; LU, B. L.; LIN, J.J.; LI, Y.H.; LEE, M.R. A method to prepare magnetic nanosilicate platelets for effective removal of microcystis aeruginosa and microcystin - LR. In: Holst $\mathbf{O}$. (eds) Microbial Toxins. Methods in Molecular Biology, v. 1600, 2017, p. 85-94. Humana Press.

CHEN, D.; ZHU, H.; WANG, X. A facile method to synthesize the photocatalyticTiO2/montmorillonite nanocomposites with enhanced photoactivity. Applied Surface Science, v. 319, 2014, p. $158-166$.

CHEN, K. Y.; CHEN, Y. W. Synthesis of spherical titanium dioxide particles by homogeneous precipitation in acetone solution. Journal of Sol-Gel Science and Technology, v. 27, 2003, p. 111117.

COUTO, R. S. P.; GUARINO, A.; BRANCO, W. S.; PALERMO, E. F. A.; AZERO, E. G. Application of Clay Minerals and Polymeric Resins to Remove Dissolved Microcystin-LR, from Water. Int. J. Environ. Res., v. 7, n. 2, 2013, p. 435-442.

CURCIO, Monique Seufitellis. Preparação de materiais poliméricos como suporte para TiO2 e aplicação na degradação fotocatalítica de Fomaldeído. Rio de Janeiro. Dissertação. Universidade Estadual do Norte Fluminense, 2011.
DENG, H.; ZHANG, Y.; GENG, S. Enhanced copper removal from aqueous solution by hydrous TiO2/zeolite composite. Advances in Applied Ceramics, v. 117, n. 2, 2018, p. 118-126.

DOGAN, M.; ALKAN, M.; KARAOGLU, M. H. Kinetic analysis of reactive blue 221 adsorption on kaolinite. Desalination, v. 256, 2010, p. 154-165.

DROGUI, P.; DAGHRIR, R.; ROBERT, D. Modified TiO2 for Environmental Photocatalytic Application: A review. Industrial \& Engineering Chemistry Research. v. 52, 2013, p. 3581-3599.

GALEANO, L. A.; FLÓREZ, M. G.; GIL, A.; SÁNCHEZ, C. A.; VICENTE, M. A. Disinfection by Chemical Oxidation Methods. Applications of Advanced Oxidation Processes in Drinking Water Treatment. v. 67, 2017, p. 257-296.

SRIKANTH, B.; GOPINATH, K. P.; GOUTHAM, R.; NARAYAN, R. B.; RAMPRASATH, A.; SANKARANARAYANAN, A. R. Recent advancements in supporting materials for immobilised photocatalytic applications in wastewater treatment. Journal of Environmental Management, v. 200, 2017, p. 60-78.

GU, N.; GAO, J.; WANG, K.; YANG, X.; DONG, W. ZnO-montmorillonite as Photocatalyst and Flocculant for Inhibition of Cyanobacterial Bloom. Water Air Soil Pollut, v. 226, 2015, p. 136-148.

GU, N.; GAO, J.; LI, H.; WU, Y.; MA, Y.; WANG, K. Montmorillonite-supported with $\mathrm{Cu} 2 \mathrm{O}$ nanoparticles for damage and removal of Microcystis aeruginosa under visible light. Applied Clay Science, v. 132-133, 2016, p. 79-89.

KAKIHANA, M.; YOSHIMURA, M. Synthesis and Characteristics of Complex Multicomponent Oxides Prepared by Polymer Complex Method. Bulletin of the Chemical Society of Japan, v. 72, n. 7, 1999, p. 1427-1443.

LABILLE, J.; HARNS, C.; BOTTERO, J. Y.; BRANT, J. Heteroaggregation of Titanium Dioxide Nanoparticles with Natural Clay Colloids. Environmental Science \& Technology, v. 49, 2015, p. 6608-6616.

LEAL, A. C.; SOARES, M. C. P. Hepatoxicidade da cianotoxina microcistina. Revista da Sociedade Brasileira de Medicina Tropical. v. 37, 2004, p. 84-89.

LEITE, E. R.; SOUZA, C. M. G.; LONGO, E.; VARELA, J. A. Influence of polymerization on the synthesis of SrTiO3: Part I: characteristics of the polymeric precursors and their thermal decomposition. Ceramics International, v. 21, 1995, p. 143-152.

LIU, I.; LAWTON, L. A.; ROBERTSON, P. K. J. Mechanistic Studies of the Photocatalytic Oxidation of Microcystin-LR: An Investigation of Byproducts of the Decomposition Process. Environmental Science \& Technology. v. 37, 2003, p. 3214-3219.

LIU, J.; ZHANG, G. Recent advances in synthesis and applications of clay-based photocatalysts: a review. Phys. Chem. Chem. Phys., v. 16, 2014, p. 8178-8192. 
LIU, S.; LIM, M.; AMAL, R. TiO2-coated natural zeolite: Rapid humic acid adsorption and effective photocatalytic regeneration. Chemical Engineering Science, v. 105, 2014, p. 46-52.

LIU, Y. L.; WALKER, H. W.; LENHART, J. J. Adsorption of microcystin-LR onto kaolinite, illite and montmorillonite. Chemosphere, v. 220, 2019, p. 606-705.

MAROTHU, V. K.; GORREPATI, M.; LELLA, R. K. C.; NAGI, F. I.; SALAH, A. M. I. Photocatalysis of b-blockers - An overview. Arabian Journal of Chemistry, 2014.

MAZYCK, D. W.; BACH, M. T.; CHESNUTT, T. E. Improvement of thermal reactivation of activated carbon for the removal of 2-methylisoborneol. Water Research, v. 41, 2007, p. 79-86.

MILLS, A.; LE HUNTE, S. An overview of semiconductor photocatalysis. Journal of Photochemistry and Photobiology A: Chemistry, v. 108, 1997, p. 1-35.

MIRANDA-GARCÍA, N.; SUÁREZ, S.; MALATO, S.; MALDONADO, M. I.; SÁNCHEZ, B. Regeneration approaches for TiO2 immobilized photocatalystused in the elimination of emerging contaminants in water. Catalysis Today, v. 230, 2014, p. 27-34.

MORRIS, R. J.; WILLIAMS, D. E.; LUU, H. A.; HOLMES, C. F. B.; ANDERSEN, R. J.; CALVERT, S. E. The adsorption of microcystin-LR by natural clay particles. Toxicon, v. 38, 2000, p. 303-308.

PESTANA, C. J.; EDWARDS, C.; PRABHU, R.; ROBERTSON, P. K. J.; LAWTON, L. A. Photocatalytic degradation of eleven microcystin variants and nodularin by $\mathrm{TiO} 2$ coated glass microspheres. Journal of Hazardous Materials, v. 300, 2015, p. 347-353.

PIERRE, A. C.; Sol-gel processing of ceramic powders. American Ceramic Society Bulletin, v. 7, 1991, p. 1281-1288.

PINHO, L. X.; AZEVEDO, J.; BRITO, A.; SANTOS, A.; TAMAGNINI, P.; VILAR, V. J. P.; VASCONCELOS, V. M.; BOAVENTURA, R. A. R. Effect of TiO2 photocatalysis on the destruction of Microcystis aeruginosa cells and degradation of cyanotoxins microcystin-LR and cylindrospermopsin. Chemical Engineering Journal, v. 268, 2015, p. 144-152.

PRONINA, N.; DENISS, K.; MOISEEV, A.; DEUBENER, J.; KRICHEVSKAYA, M. Titanium dioxide sol-gel-coated expanded clay granules for use in photocatalytic fluidized-bed reactor. Applied Catalysis B: Environmental, v. 178, 2015, p. 117-123.

RAHMAN, A.; NURJAYADI, M.; WARTILAH, R.; KUSRINI, E.; PRASETYANTO, E. A.; DEGERMENCI, V. Enhanced activity of TiO2/natural zeolite composite for degradation of methyl orange under visible light irradiation. International Journal of Technology, v. 6, 2018, p. 1159-1167.

RAMÍREZ, J. H.; GALEANO, L. A. Natural Organic Matter Removal by Heterogenous Catalytic Wet Peroxide Oxidation (CWPO). Applications of Advanced Oxidation Processes in Drinking Water Treatment, v. 67, 2017, p. 69-98.
RUBIO, D.; CASANUEVA, J. F.; NEBOT, E. Improving UV seawater disinfection with immobilized TiO2: Study of the viability of photocatalysis (UV254/TiO2) as seawater disinfection technology. Journal of Photochemistry and Photobiology A: Chemistry. v. 271, 2013, p. 16-23.

SONG, J.; WANG, X.; MA, J.; WANG, X.; WANG, J.; XIA, S.; ZHAO, J. Removal of Microcystis aeruginosa and Microcystin-LR using a graphitic- C3N4/TiO2 floating photocatalyst under visible light irradiation. Chemical Engineering Journal, v. 348, 2018, p. 380388.

STEFAN, M. I. Advanced Oxidation Processes for Water Treatment: Fundamentals and Applications. IWA Publishing, 2018.

SUN, Q.; HU, X.; ZHENG, S.; SUN, Z.; LIU, S.; LI, H. Influence of calcination temperature on the structural, adsorption and photocatalytic properties of $\mathrm{TiO} 2$ nanoparticles supported on natural zeolite. Powder Technology, v. 274, 2015, p. 88-97.

SZCZEPANIK, B. Photocatalytic degradation of organic contaminants over clay-TiO2 nanocomposites: A review. Applied Clay Science, v. 141, 2017, p. 227-239.

TADOKORO, S. K.; MUCCILO, E. N. S. Zircônia tetragonal policristalina. Parte 1: Síntese e caracterização. Cerâmica, v. 46, n. 300, 2000, p. 230-237.

TCHOBANOGLOUS, G.; STENSEL, H. D.; TSUCHIHASHI, R.; BURTON, F.; ABU-ORF, M.; BOWDEN, G.; PFRANG, W. Metcalf \& Eddy. Tratamento de efluentes e recuperação de recursos. Tradução: Ivanildo Hespanhol, José Carlos Mierzwa. 5 ed. Porto Alegre, AMGH, 2016. 1980p.

VAIZOGULLAR, A. I. TiO2/ZnO Supported on sepiolite: preparation, structural characterization, and photocatalytic degradation of Flumequine Antibiotic in aqueous solution. Chemical Engineering Communications, v. 204, 2017, p. 689-697.

WANG, B.; GODOI, F. C. de; SUN, Z.; ZENG, Q.; ZHENG, S.; FROST, R. L. Synthesis, characterization and activity of an immobilized photocatalyst: Natural porous diatomite supported titania nanoparticles. Journal of Colloid and Interface Science, v. 438, 2015, p. 204-211.

WANG, B.; ZHANG, G.; SUN, Z.; ZHENG, S. Synthesis of natural porous minerals supported $\mathrm{TiO} 2$ nanoparticles and their photocatalytic performance towards Rhodamine B degradation. Powder Technology, v. 262, 2014, p. 1-8.

WANG, F.; GAO, Y.; SUN, Q.; MEGHARAJ, M.; NAIDU, R.; CHEN, Z. Degradation of microcystin-LR using functional clay supported bimetallic Fe/Pd nanoparticles based on adsorption and reduction. Chemical Engineering Journal, v. 225, 2014, p. 55-62.

WANG, X.; WANG, X.; ZHAO, J.; SONG, J.; WANG, J.; MA, R.; MA, J. Solar light-driven photocatalytic destruction of cyanobacteria by F-Ce-TiO2/expanded perlite floating composites. Chemical Engineering Journal, v. 320, 2017, p. 253-263. 
WANG, Y.; CHEN, C.; GUTEN, U. V.; QU, Q.; YAO, W.; YU, G. Comparison of methylisoborneol and geosmin abatement in surface water by conventional ozonation and an electro-peroxone process. Water Research, v. 108, 2017, p. 373-382.

XING, Z.; ZHOU, W.; CUI, J.; KUANG, J.; WAN, N.; XIU, Z.; YIN, J.; ZHANG, J.; ZHAO, T. Recent advances in floating TiO2-based photocatalysts for environmental application. Applied Catalysis B: Environmental, v. 225, 2018, p. 452-467.
ZHANG, G.; SONG, A.; DUAN, Y.; ZHENG, S. Enhanced photocatalytic activity of TiO2/zeolite composite for abatement of pollutants. Microporous and Mesoporous Materials, v. 255, 2018, p. 61-68.

ZOU, X.; WAN, Z.; WAN, C.; ZHANG, G.; PAN, X.; PENG, J.; CHANG, J. Novel $\mathrm{Ag} / \mathrm{AgCl} / \mathrm{K} 6 \mathrm{Nb} 10.8030$ photocatalyst and its enhanced visible light photocatalytic activities for the degradation of microcystin-LR and acid red G. Journal of Molecular Catalysis A: Chemical, v. 411, 2016, p. 364-371. 